\title{
A Degenerate Case of the Timoshenko Beam Prob- lem with Boundary Damping: An Asymptotic and Numerical Study
}

\author{
Matthew P. Coleman \\ Department of Mathematics and Computer Science, Faireld University, Faireld, CT 06824
}

\author{
Les Schaffer \\ Department of Physics, Faireld University, Faireld, CT 06824
}

\begin{abstract}
(Received 24 December 2010, accepted 3 August 2011)
Recently, the Timoshenko beam, and generalizations thereof, have been considered as models for the vibration of single and multiple walled carbon nanotubes. Further, it has been shown that, in some instances, carbon nanotubes and nanotube sheets possess the common property that their Poisson's ratio is negative. In these cases, the degenerate case of the Timoshenko beam problem, where $E=k^{\prime} G$, becomes physically realizable. The asymptotic derivation of the vibration spectrum in this case is qualitatively different from that for the nondegenerate case. We performed such a derivation, and we compared the asymptotic results with numerical results. Further, we performed parameter studies based on the exact frequency equation for the undamped case for values of $E / k^{\prime} G$ on intervals including $E / k^{\prime} G=1$. The results show very interesting and complex behavior throughout a fairly large interval about $E / k^{\prime} G=1$.
\end{abstract}

\section{INTRODUCTION}

The asymptotic estimation of the vibration spectrum of the dissipative Timoshenko beam problem with constant coefficients was performed in Coleman and Wang ${ }^{1}$ and generalized to the case of $\mathrm{x}$-dependent coefficients in Shubov. ${ }^{2}$ However, in each case, the computations were performed under the assumption that $E \neq k^{\prime} G, E I / K \neq I_{\rho} / \rho$, where $\rho$ is the linear mass density; $E$, Young's modulus; $I$, the area moment of inertia for bending; $I_{\rho}$, the mass moment of inertia; $G$, the shear modulus; $k^{\prime}$ the shape factor; and $K$ the shear stiffness. Indeed, the arguments therein fail if we set $E I / K=I_{\rho} / \rho$ from the start.

Here, then, we treat the degenerate case when the coefficients are constant. The asymp- totic computations and, in particular, the reflection coefficients, are seen to be qualitatively different from those for the case $E I / K \neq I_{\rho} / \rho$. As for the results, we again see the typical double-branched Timoshenko spectrum, with each branch corresponding to its own damping rate determined by one of the two damping parameters. What is interesting, although not surprising, is that the eigenfrequencies now appear in pairs with equal imaginary parts, one for each branch. Thus, for the special case involving conservative boundary conditions, the two branches merged into one "double-branch" asymptotically.

Further, we investigated the spectrum's behavior as $E I / K \rightarrow I_{\rho} / \rho$. Our numerical results suggest that the nondegenerate spectrum approaches the degenerate spectrum smoothly, at least in an asymptotic sense-indeed, if we set $E I / K=I_{\rho} / \rho$ in the asymptotic formulas in Coleman and Wang $^{1}$ and Shubov, ${ }^{2}$ the results match those given here (although, as we show, the convergence for the degenerate case is slower than for the non-degenerate). However, we also performed parameter studies for the exact frequency equation for the undamped beam, and these show that the spectrum possesses a much richer and more interesting behavior than is suggested by the asymptotic and numerical results.

An important consideration is whether the criterion $E I / K=I_{\rho} / \rho$ is physically realizable. We have that $I_{\rho}=\sigma I$ and $K=k^{\prime} G A$, where $\sigma$ is the mass per unit volume and, $A$ the cross-sectional area. Further, $E=2(1+\nu) G$, where $\nu$ is the Poisson's ratio. Thus,

$$
\frac{E I}{K}=\frac{I_{\rho}}{\rho} \Longleftrightarrow k^{\prime} G,
$$

from which it follows that

$$
2(1+\nu)=k^{\prime}
$$

or

$$
\gamma^{2}=\frac{E}{k^{\prime} G}=\frac{2(1+\nu)}{k^{\prime}}=1 .
$$

For standard materials, this is impossible, as $0<\nu<0.5$ and $0<k^{\prime}<1$.

There are materials, known as auxetics, that possess a negative Poisson's ratio. Although many of these materials seem to be ill suited to the design of beam-like objects, there are exceptions. In particular, the field of nanotechnology has exploded onto the scene in recent years, and there is great interest in the study of carbon nanotubes, given their many desirable properties. While their mechanical behavior has been studied via models based on molecular dynamics, these models can be fairly complex and cumbersome, and the computations timeconsuming. Thus, people have explored alternative models, and there is surprising evidence that models from continuum mechanics may give satisfactory results even for structures only a few atoms thick. ${ }^{3,4}$ In particular, the Euler-Bernoulli and Timoshenko beam equations have been used to model the 